\title{
Mesoscale Laser Processing using Excimer and Short-pulse Ti:Sapphire Lasers
} (M702)

M. D. Shirk, A. M. Rubenchik, G. H. Gilmer, B. C. Stuart, J. P. Armstrong, S. K. Oberhelman, S. L. Baker, A. J. Nikitin, R. P. Mariella Jr., Lawrence Livermore National Laboratory, Livermore, CA

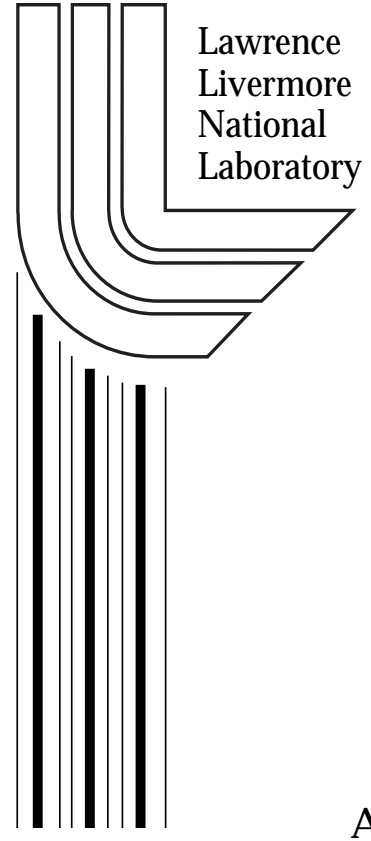

This article was submitted to

ICALEO, Jacksonville, Florida, October 13-16, 2003

July 28, 2003 


\section{DISCLAIMER}

This document was prepared as an account of work sponsored by an agency of the United States Government. Neither the United States Government nor the University of California nor any of their employees, makes any warranty, express or implied, or assumes any legal liability or responsibility for the accuracy, completeness, or usefulness of any information, apparatus, product, or process disclosed, or represents that its use would not infringe privately owned rights. Reference herein to any specific commercial product, process, or service by trade name, trademark, manufacturer, or otherwise, does not necessarily constitute or imply its endorsement, recommendation, or favoring by the United States Government or the University of California. The views and opinions of authors expressed herein do not necessarily state or reflect those of the United States Government or the University of California, and shall not be used for advertising or product endorsement purposes.

This is a preprint of a paper intended for publication in a journal or proceedings. Since changes may be made before publication, this preprint is made available with the understanding that it will not be cited or reproduced without the permission of the author.

This report has been reproduced directly from the best available copy.

Available electronically at http://www.doc.gov/bridge

Available for a processing fee to U.S. Department of Energy

And its contractors in paper from

U.S. Department of Energy

Office of Scientific and Technical Information

P.O. Box 62

Oak Ridge, TN 37831-0062

Telephone: (865) 576-8401

Facsimile: (865) 576-5728

E-mail: reports@adonis.osti.gov

Available for the sale to the public from

U.S. Department of Commerce

National Technical Information Service

5285 Port Royal Road

Springfield, VA 22161

Telephone: (800) 553-6847

Facsimile: (703) 605-6900

E-mail: orders@ntis.fedworld.gov

Online ordering: http://www.ntis.gov/ordering.htm

OR

Lawrence Livermore National Laboratory

Technical Information Department's Digital Library

http://www.llnl.gov/tid/Library.html

Approved for public release; further dissemination unlimited 
Mesoscale Laser Processing using Excimer and Short-pulse Ti:Sapphire Lasers (M702)

M. D. Shirk, A. M. Rubenchik, G. H. Gilmer, B. C. Stuart, J. P. Armstrong, S. K. Oberhelman, S. L. Baker, A. J.

Nikitin, R. P. Mariella Jr., Lawrence Livermore National Laboratory, Livermore, CA

\begin{abstract}
Targets to study high-energy density physics and inertial confinement fusion processes have very specific and precise tolerances that are pushing the state-of-the-art in mesoscale microsculpting technology. A significant effort is required in order to advance the capabilities to make these targets with very challenging geometries. Ultrashort pulsed (USP) Ti:Sapphire lasers and excimer lasers are proving to be very effective tools in the fabrication of the very small pieces that make up these targets. A brief description of the dimensional and structural requirements of these pieces will be presented, along with theoretical and experimental results that demonstrate to what extent these lasers are achieving the desired results, which include sub- $\mu \mathrm{m}$ precision and RMS surface values well below 100 $\mathrm{nm}$. This work indicates that excimer lasers are best at sculpting the polymer pieces and that the USP lasers work quite well on metal and aerogel surfaces, especially for those geometries that cannot be produced using diamond machining and where material removal amounts are too great to do with focused ion beam milling in a cost effective manner. In addition, the USP laser may be used as part of the procedure to fill target capsules with fusion fuel, a mixture of deuterium and tritium, without causing large perturbations on the surface of the target by keeping holes drilled through $125 \mu \mathrm{m}$ of beryllium below $5 \mu \mathrm{m}$ in diameter.
\end{abstract}

\title{
1. INTRODUCTION
}

The manufacture of laser targets for the study of high-energy density physics (HEDP) and inertial confinement fusion (ICF) exhibit some challenges to the state of the art in mesoscale forming processes. The goal of processes such as inertial-confinement fusion is to uniformly compress and heat the fuel, a mixture of deuterium and tritium (DT), to cause fusion [1]. HEDP studies physics analogous to events such as supernova [2], except the experiments are performed on a $\mu \mathrm{m}$ to millimeter scale, instead of normal macroscopic dimensions. Non-uniform compression caused by inhomogeneity of either the laser beam or perturbations at surface interfaces in the targets are analogous to holding a large ball of dough in your hand and squeezing. Instead of compressing it, much of it slips out through your fingers. These targets are used in an effort to study the effects of perturbations that induce instabilities [3, 4]. Experiments, which are being performed currently and are proposed for the future, demand much higher precision machining of novel materials than has been demonstrated with conventional processing techniques - for example, adding $\mu \mathrm{m}$-scale dimples on the inside of a 1-mm diameter hemispherical shell. Figure 1 shows a copper surface with a field of moguls machined on the surface that was done by this group about 2 years ago. It has generated much interest in the target fabrication community.

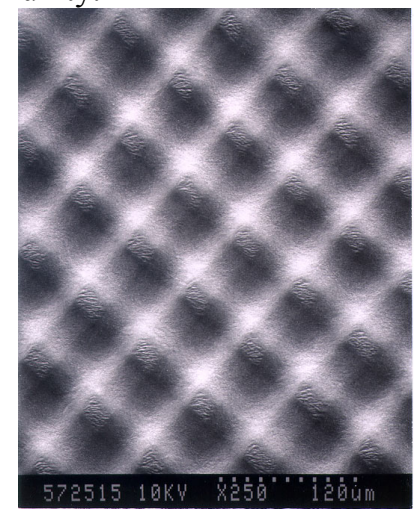

Figure 1. A picture of a field of moguls, left behind after a polished copper surface has been patterned with laser ablation, serves as an early demonstration of the abilities of USP lasers to generate a surface with the many of the qualities that the HEDP researchers desire.

The use of USP and excimer lasers in target fabrication is an enabling tool in the study of ICF and HEDP. These lasers allow the fabrication of targets with very precise control of features on the surfaces of parts. Technologies are being developed to manufacture mesoscale targets ( $\sim 1$ to 3-mm diameter cylinders and spheres) that have multiple small pieces made out of various materials including metals, polymers, ceramics, carbon foams, and metal-oxide aerogel materials with surfaces machined to finishes of less than 100-nm RMS roughness and 
precisely controlled surface features. These will be shaped using a combination of focused ion beam (FIB) milling, fast-servo diamond machining, and laser ablation. All three of these technologies are necessary for the manufacture of these targets, as they complement each other.

FIB is a relatively slow and expensive process that can perform extremely accurate material removal, but if a cut is not completely through a material, then ions are left embedded in the material, and the material's microstructure is often modified downstream of the removed material. Fast-servo diamond machining is both precise and relatively cost effective, but can handle only certain materials and geometries. USP lasers and excimer lasers can fill in the gaps by handling both difficult geometries and exotic materials, in a manner that is faster and less costly than FIB. They can also add value to diamond machined surfaces by performing laser smoothening to reduce the surface roughness of a diamond machined surface.

In addition to surface sculpting and smoothening, lasers are being used to drill and seal the fill holes in beryllium ICF targets so that high-pressure deuterium-tritium fuel can be used for ICF experiments. It is critical that the process of drilling, filling, and sealing the capsule be as minimally perturbing as possible. In thinner plastic targets, especially at lower pressure, the targets can be filled by using diffusion processes. For metals such as beryllium, where the material will be withstanding higher internal pressures, it is necessary to have a sealable hole for filling the target with fuel. To minimize the scar left on the surface, very small holes and sealing welds are necessary. We can consistently drill holes of approximately 2 to $3-\mu \mathrm{m}$ diameter through $125 \mu \mathrm{m}$ of beryllium, and are working on techniques to seal them in a high pressure DT atmosphere.

A discussion of the present theory on how these lasers interact with surfaces will be presented. This will be followed with experimental methods to demonstrate the current state of the art in laser micro-sculpting and smoothening in plastics, metals, and aerogels. Small hole drilling in metals will also be presented.

\section{EXPERIMENT AND MODELING METHODS}

Experimental studies have been performed using both 100-fs class Ti:Sapphire lasers and 193-nm, 25-ns ArF Excimer lasers. These studies have been complemented and enhanced by two different theoretical methods using both hydrocode models and molecular dynamics (MD) simulations to study surfaces under laser irradiation. The focus of the modeling effort has been on the short-pulse interactions because they are newer and less studied than those with nanosecond pulse duration.

\subsection{Computational studies}

\subsubsection{Hydrocode model of laser ablation and smoothening}

The studies of material ablation by the short laser pulses consider the scales of materials modification and ejecta that are much smaller than the laser spot, thus a one-dimensional treatment of the problem is adequate.

A one-dimensional radiation hydrocode HYADES [5] was used which models material as electron and ion fluids. The hydrodynamic equations are solved in Lagrangian coordinates, which means that the computational mesh is 'frozen' into the fluid and moves with it: this method is well suited for the materials which may undergo density changes of several orders of magnitude during the simulations. This code includes a wave solver for the description of laser interaction with material. As a result, the code takes into account the change of the absorption during the pulse due to the change of electron density, spatial profile and temperature. It is essential to include these effects when modeling the interaction of femtosecond laser pulses with solids.

The equation of state (EOS) quantities and related thermodynamics coefficients are obtained from external tables [6] that have been formed using experimental data and /or theoretical models. For the interaction with metals we used Thomas-Fermi model.

Material electromagnetic properties are described with an extended Drude model that includes both intraband and interband transitions.

The description of thermal transport includes temperature-dependent thermal conduction and heat capacities. Thermal conduction is determined by the collision frequency in the Drude model. The cold value of transport constants are matched with experimental data and interpolated to the hot plasma expression for ideal plasma. The description takes into account the change of transport coefficients at melting point and pressure-induced shift of melting point according the Lindemann law. The code was benchmarked by modeling the measured absorption of ultra-short pulses in aluminum for a wide range of wavelength and intensities.

\subsubsection{MD model of laser ablation and smoothening in metals}

Laser ablation was modeled using large-scale molecular dynamics (MD) simulations of single-crystal copper. The computational cells contained from four to thirty million atoms. The parallel LLNL code MDCASK used in these simulations is based on classical dynamics and MPI message passing routines. Interactions between the copper atoms were simulated using the embedded atom (EAM) potential of Mishin [7]. Periodic boundary conditions were applied in the two lateral directions of the computational cell. In the cell direction aligned with the 
laser beam, an open boundary was implemented on the end corresponding to the free surface of the copper, which allows the ablated material to leave the cell. At the other end of the cell, an absorbing boundary condition was implemented to suppress reflections of the shock wave produced by the ablation [8]. The lateral extent of the computational cells, up to $65 \mathrm{~nm}$, is much smaller than the lateral extent of the laser beam. The configurations formed during ablation, although much smaller than the width of the beam, are large compared to traditional MD simulations, and require large computer clusters with fast microprocessors to provide useful information. This work was performed on a Linux cluster with over 2,000 Intel 2.4-GHz processors. Several days were required to complete a run with 30 million atoms covering a period of about $200 \mathrm{ps}$.

The interaction of the femtosecond laser pulse with the copper involves the initial transfer of electro-magnetic energy to the electrons, and then from the electrons to the copper ions. The EAM potential does not account for effects of electronic excitations during irradiation or the electronic contribution to the thermal conductivity. Electrons are included only to the extent that they account for the interatomic forces. For this reason, we also employ the one-dimensional code HYADES [5]. It is used to model the first picosecond after the beginning of the laser pulse, during which time the major part of the electronic energy is transferred to the copper atoms. The properties of the copper obtained from HYADES at the end of this time are used as initial conditions for the MD simulation. In addition, HYADES is used to correct the temperature distribution during the run, in this way accounting for the electronic conductivity.

These large-scale MD simulations with tens of millions of atoms have been performed to simulate the ablation process in copper. However, the experiments discussed below demonstrate that the ablation process generates structures with sizes up to several tenths of a micrometer for laser pulse energies near the ablation threshold energy. A MD simulation of a cube with $0.5 \mu \mathrm{m}$ edges would require more than 10 billion atoms, a simulation at the borderline of feasibility. Although the generation of shock waves, material removal, and other phenomena are accurately simulated using systems smaller than these observed structures, our interest in the formation of the surface and its relationship to the nucleation and growth of large voids and ejected particles requires methods obtain data on the physical length scale. Previous workers have used techniques such as simulating the trajectories of particles instead of individual atoms; such particles that represent groups of atoms are the fundamental unit of the simulations [9]. This approach, which eliminates the need for complex parameterization of the particles, has been to develop a scaling relationship between the length scale of the structural features and with the length corresponding to the depth distribution of the heat imparted to the ions. This distribution obtained from HYADES can be approximated as an exponential decay, $\exp (-\mathrm{z} / \lambda)$, where $\lambda=100 \mathrm{~nm}$. In order to treat the surface features in reduced scale, we have done simulations at $\lambda$ values of 11 and $22 \mathrm{~nm}$. We find that the feature sizes are approximately linear in $\lambda$.

\subsection{Experimental method}

\subsubsection{Laser experimental setups}

A Lambda-Physik Model LPX 300 ArF excimer laser was used to ablate various targets using mask projection imaging optics. Experimental Layout is in Figure 1. The samples were positioned on a servo-driven, $x-y$ table with approximately $1-\mu \mathrm{m}$ accuracy and controlled by a MM4005 motion controller. Samples were held to the stage using a vacuum chuck. Software was written in Labview to perform velocity profiling to form features such as moguls and sinusoidal waves.

Both Ti:Sapphire lasers used in this work are systems built at Lawrence Livermore National Laboratory (LLNL). The first operates at $1-\mathrm{kHz}$, with a maximum energy of approximately $2 \mathrm{~mJ}$ per pulse with a wavelength of $820 \mathrm{~nm}$, with 120-fs pulses. This laser's repetition rate was often reduced to $100 \mathrm{~Hz}$, using the on/off pockels cell after the regenerative amplifier to make delivery of a small number of pulses easier. This laser was used for direct surface sculpting. The important components for controlling laser power include a combination of a 1/2 waveplate and the compression grating, which also works as a polarizer, pockels cell (slicer) to control repetition rate and minimize pre-pulse due to round-trip leakage, and delivery optics (lenses, $1 / 4$ waveplate or $1 / 2$ waveplate, flat mirrors), galvo-scanners, and a vacuum chamber that operates at $10 \mathrm{mTorr}$ ( 1 Torr $=133$ pascals), as shown in Figure 2. A CCD camera is used on the input side of the chamber to view the workpiece as the surface is sculpted. The final focus lens is also used as the objective lens in the telescope that images the surface onto the CCD camera. A 2nd CCD camera is placed after the chamber to do beam diagnostics. In order to set up this diagnostic, the final focus lens is removed and a reticle is placed on the same plane that the workpiece will be placed. The reticle is then imaged unfocused laser light onto the CCD camera after the chamber with approximately $4 x$ optical magnification onto the CCD chip. Light intensity is controlled by neutral-density filters. The reticle is then illuminated with white light from the back and imaged onto the CCD camera before the chamber so that this can be used to bring the workpiece to the correct location so that the well-characterized spot can be used for sculpting. 


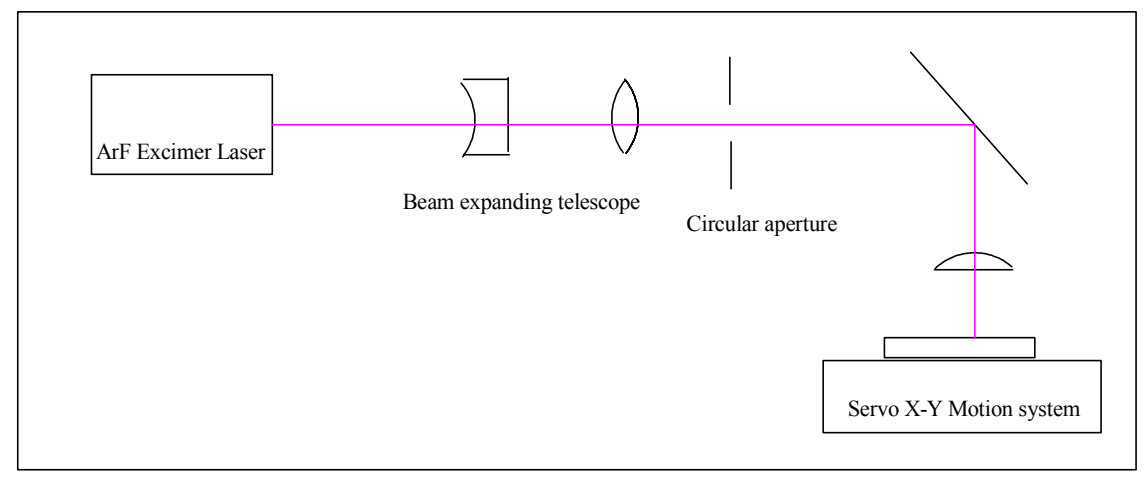

Figure 2. Experimental layout of excimer laser experiments.

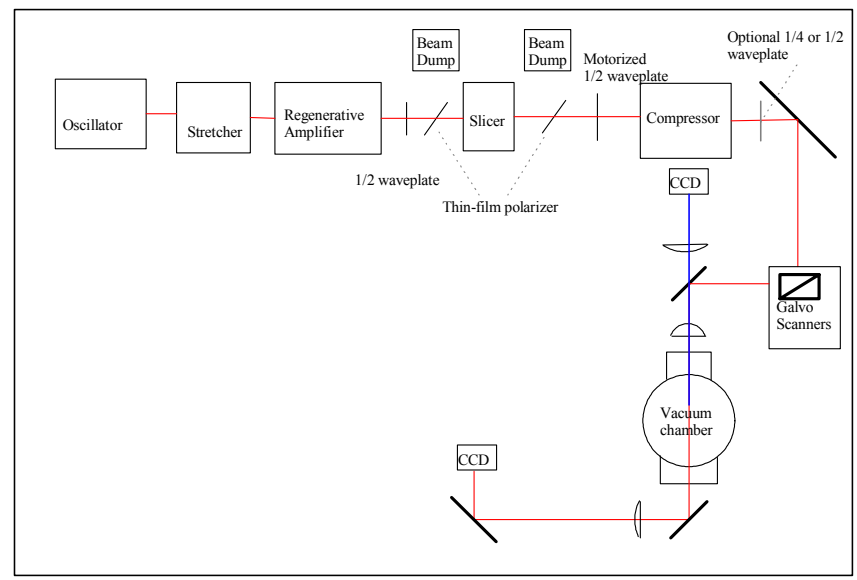

Figure 3. Experimental layout of the USP Ti:Sapphire laser surface sculpting apparatus.

The 2nd Ti:Sapphire laser was used for the small hole drilling efforts. It operates at $3.5 \mathrm{kHz}$, with maximum pulse energy of $750 \mu \mathrm{J}$, at $810 \mathrm{~nm}$ and $120 \mathrm{fs}$. These pulses are then frequency converted to $405 \mathrm{~nm}$. This is then delivered through an optical system into a vacuum chamber as shown in Figure 3. The optical delivery of the pulses was accomplished by delivering the pulses into the collimated space of an infinite-conjugate-ratio microscope. The objective chosen for this project was a single-element aspheric lens with a focal length of $4 \mathrm{~mm}$ and a clear aperture of $4.6 \mathrm{~mm}$, yielding a numerical aperture of 0.58 . The lens is housed in the vacuum chamber and is protected by a microscope coverslip.

Due to the infinite conjugate designed system, the focal plane of the laser and the object plane of the microscope are at the same location. This allows the operator to accurately place the target and view the hole as it is drilled. The microscope itself consists of a fiber-delivered laser illumination source, the aspheric objective lens, and a $40-\mathrm{cm}$-focal-length tube lens to bring the image created by the objective to the plane of a CCD camera. The field of view of this camera is $75 \mu \mathrm{m}$ with a resolution of about $200 \mathrm{~nm}$ per pixel. 


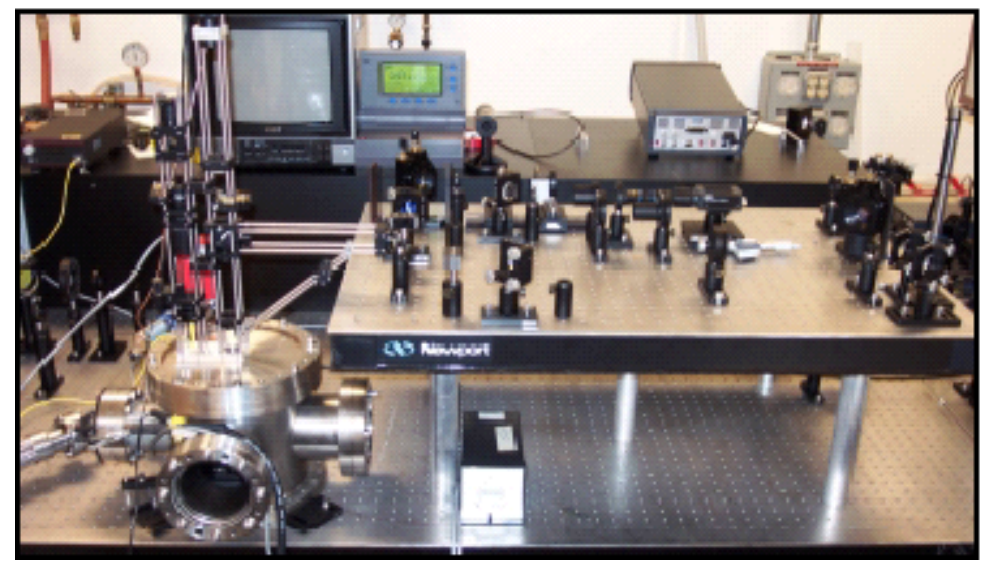

Figure 4. A photograph of the NIF small hole drilling station.

\subsubsection{Materials}

Two metals were used in the surface sculpting studies. The first included 4 pieces of pure copper. One polycrystalline, one (111) surfaced single crystal, one (110) surfaced single crystal, and one (100) surfaced single crystal. Laser machining was also performed on 1/2" diameter electroless Ni Plated witness samples from Corning Netoptix in St Keene New Hampshire that were diamond turned to optical flatness (typical surface roughness was 15 $\mathrm{nm}, \mathrm{RMS}$ ). The titania aerogel samples were made at LLNL to $1.8 \mathrm{~g} / \mathrm{cm}^{3}$ density by Satcher [10] Samples were characterized by optical profilometry, scanning electron microscopy, and atomic-force microscopy (AFM), before and after laser treatment.

Beryllium capsules and foils were made at LLNL specifically to act as surrogates for future ICF targets. The capsules were formed by sputter coating beryllium onto a $\mathrm{CH}$ polymer mandrel [11].

\subsubsection{Metrology}

The atomic force microscopy (AFM) measurements presented here were conducted on a Digital Instruments AFM. The AFM was fitted with the following components: Dimension 5000, equipped with an acoustic hood, type G scanner and a Nanoscope IIIA controller with phase extender box. The measurements were performed using Tapping Mode ${ }^{\mathrm{TM}}$ which measures topography in air by tapping the surface with an oscillating probe tip. The probe tips were etched silicon with a nominal tip radius of 5 to $10 \mathrm{~nm}$.

The Zygo Newview 100 is based on scanning white light interferometry, a technique in which a pattern of bright and dark lines (fringes) result from an optical path difference between a reference and a sample beam. Incoming light is split inside an interferometer, one beam going to an internal reference surface and the other to the sample. After reflection, the beams recombine inside the interferometer, undergoing constructive and destructive interference and producing the light and dark fringe pattern. In the Newview 100, a precision vertical scanning transducer and camera together generate a three dimensional interferogram of the surface, processed by the computer and transformed by frequency domain analysis resulting in a quantitative 3-D image. The 3-D areal surface measurements have a vertical resolution of $0.1 \mathrm{~nm}$ and a lateral resolution (objective dependent) of $1.0 \mu \mathrm{m}$.

The high-resolution micrographs shown here were taken with a LEO 1560 scanning electron microscope (SEM) equipped with a Gemini field emission column and an in-lens annular detector. The LEO 1560 is capable of resolving 1-nm objects at a voltage of $20 \mathrm{kV}$ and $3-\mathrm{nm}$ objects at $1 \mathrm{kV}$.

\section{RESULTS AND DISCUSSION}

\subsection{Computer modeling results}

Molecular dynamics calculations of laser ablation have been applied to several metals, including $\mathrm{Cu}$ and $\mathrm{Al}$. In essentially all cases, appreciable material is ablated only when the pulse energy is sufficient to melt several nm deep into the metal. The rapid heating causes thermal expansion which compresses the neighboring regions, launches a propagating compression wave deeper into the material, and gives outward momentum to material near the surface. The pressure changes from compressive to tensile as the momentum of the material near the surface carries it beyond the point of zero stress. This process causes tensile stress in the underlying liquid. HYADES calculations of the pressure wave in $\mathrm{Cu}$ are shown in Figure 5. The tensile stress in the surface region is clearly seen, although the stress approaches zero at the surface because of the requirement of continuity at the liquid/vacuum boundary. 


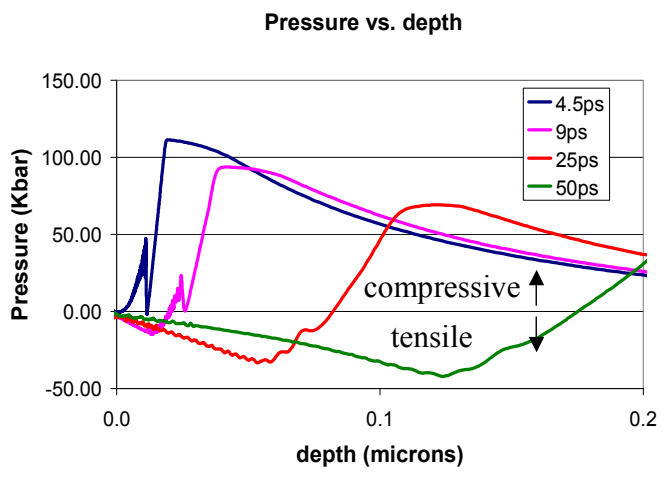

Figure 5. HYADES simulation of a 100-fs, 800 -nm laser pulse on copper. The pressure as a function of depth is plotted at several times after the initiation of the pulse.

Figure 6 shows ablation with a pulse that is $80 \%$ above threshold. The temperature of the surface just after irradiation rises to values within several hundred degrees of the liquid-vapor critical temperature of copper. As a result, the surface tension between liquid and vapor phases is extremely small and has very little stabilizing influence on the liquid phase. Many small voids form quickly, and progressively form larger voids. Incomplete merger of the voids results in liquid filaments connected to the copper base metal, but these filaments break as they are stretched, and Rayleigh instabilities cause them to pinch-off. The resulting free segments of these filaments collapse and form nearly spherical $\mathrm{nm}$-scale drops under the influence of surface tension. Some of the droplets are moving back toward the copper block, as a result of surface tension forces that occurred while they were still connected to it. Mounds with aspherical tops are observed to form when these droplets freeze on the ablated surface. These will also be observed in micrographs in the following section on experimental results on copper.
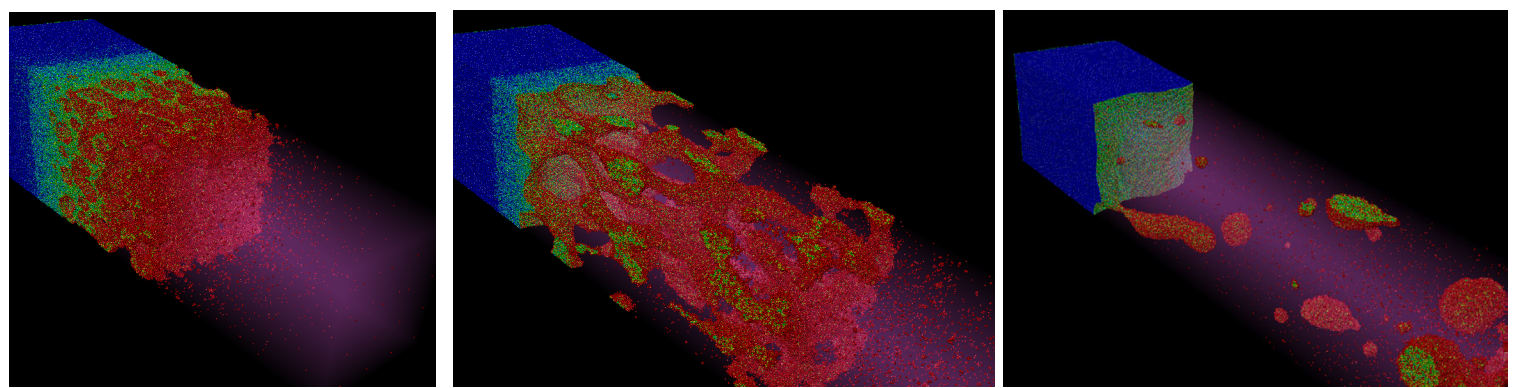

Figure 6. Molecular dynamics of laser ablation of copper, with a pulse energy $80 \%$ above threshold (left $16 \mathrm{ps,}$ center $49 \mathrm{ps}$, right $184 \mathrm{ps}$ ). The energy was imparted to the system, over a period of $100 \mathrm{fs}$, with a depth distribution of the energy that approximated an exponential decay, $\exp (-\mathrm{z} / \lambda)$, where $\lambda=11 \mathrm{~nm}$. The lateral dimensions of the sample is $32 \mathrm{~nm}$.

In conclusion, is the remaining surface roughness on a surface is a consequence of the voids that nucleate resulting from the tensile stress, and of the filaments of liquid that may freeze in an elongated shape, or if they remain liquid they can assume a spherical shape and deposit on the ablated surface as liquid droplets. The high thermal conductivity of copper causes the ablated surface region to freeze quickly, with little time for smoothening while in the liquid state. Ablation of materials of lower conductivity such as nickel tends to leave smoother surfaces, and this can be explained by the longer persistence time of liquid near the surface.

\subsection{Materials processing results}

\subsubsection{Surface sculpting}

Laser surface sculpting was performed on various materials including copper, nickel, polyimide, and titania aerogel. The excimer created superior surface finishes on the polyimide. The USP system performed better on metals and was the only system used on the aerogels. Shallow pits were machined by using a few pulses with a static beam and target. These experiments were performed on the copper, nickel, polyimide and aerogel targets. USP pulses on polyimide left a very rough, disrupted surface not worthy of further investigation since excimer ablation of polymers is already well understood and hard to improve upon using infrared pulses. Experiments were 
then performed to machine grooves onto a surface by scanning the beam using galvo-scanners or by translating the part with a motion system. These were performed with USP laser on the metal and with the excimer system on the polyimide surface.

Experiments on the copper surfaces showed that crystal orientation had minimal affect on the machining. The effects of various laser parameters including fluence $\left(\mathrm{J} / \mathrm{cm}^{2}\right)$, pulse number, and polarization (linear, circular) were observed. The depth profiles and the structures of the surface were characterized after the experiments. Figures 7 and 8 show copper surfaces that were ablated using varying number of pulses. These two pits are characteristic of all the samples and agree closely with structures observed in the modeling of copper, that being the structures seen are consistent with the freezing in of the bubbles observed in the simulation. Although there is a vast difference in the number of pulses delivered, the microstructure of the material does not change significantly. Periodic structures observed are the result of the linear polarization of the beam. On surfaces where there are scratches, or pre-existing periodicity, near-threshold ablation enhances pre-existing periodicity, whereas the final surface morphology in highenergy-density areas (significantly above threshold) transitions to instabilities caused by polarization of the laser beam.

Nickel was another material that was ablated in similar experiments, except the samples were polycrystalline films deposited on aluminum that were diamond turned to optical flatness. Single location studies were performed in a manner similar to the copper experiments. This was followed by using the galvo-scanners to machine trench structures onto the surfaces by the moving beam.
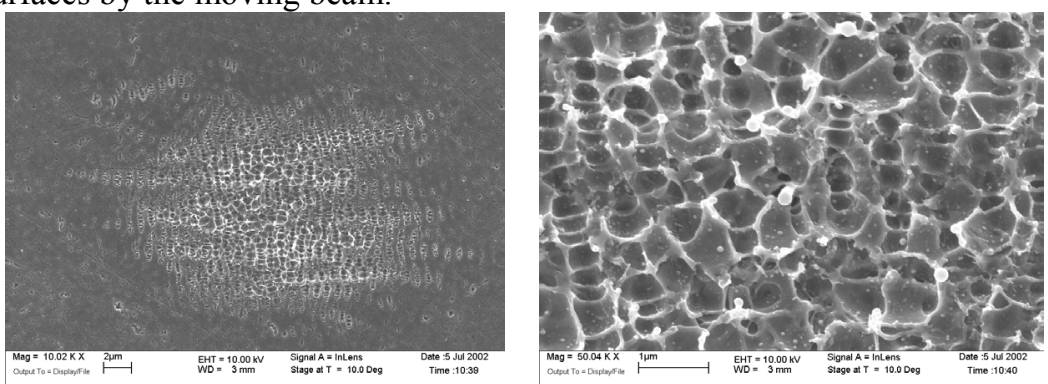

Figure 7. Copper (100) surface ablated with 5 pulses at $250 \mathrm{~mJ} / \mathrm{cm}^{2}$
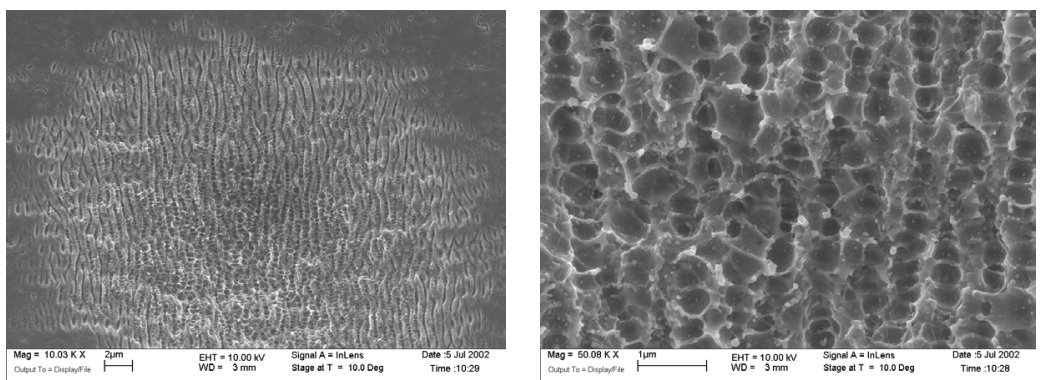

Figure 8. Copper (100) surface ablated with 100 pulses at $250 \mathrm{~mJ} / \mathrm{cm}^{2}$

Shallow pits ablated in nickel, as shown in Figure 9, show that it can leave a much smoother surface than copper. Nickel ablated by a Gaussian beam at energy densities slightly above the ablation threshold shows a range of behavior. In the center of spot, the surface is smooth to within a few nm, RMS, (smoother than the original, diamond-turned surface), with only a few nanoparticles of nickel adhering to the surface. These types of features are observed in the simulations that were performed. Moving out radially transitions to instabilities that are formed as a result of the polarization of the beam. At the perimeter of the laser spot, the diamond turned surface heterogeneously nucleates instabilities. The smooth, post-ablation surface morphology occurs in nickel and not in copper mainly because of the poorer thermal conduction of nickel. Nickel stays liquid longer, so that it can smoothen itself via the effect of "surface tension". This spurs our hopes that laser smoothening will be possible using these tools. Trenches were also ablated in nickel, and the results in all trenches that we made, some over a $\mu \mathrm{m}$ deep, were with RMS surface roughness less than $\sim 60 \mathrm{~nm}$. 

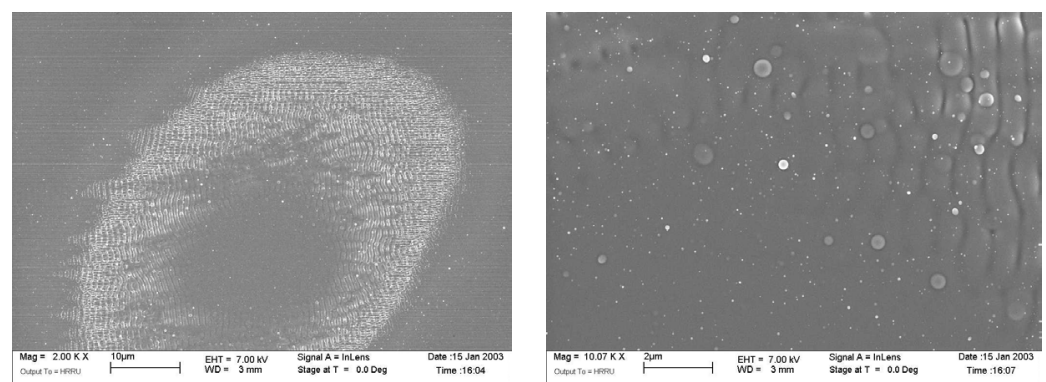

Figure 9. Nickel ablated by five $100-\mathrm{fs}, 810-\mathrm{nm}$ laser pulses at $100 \mathrm{~mJ} / \mathrm{cm}^{2}$. Left shows very similar morphology to the copper. Note how the diamond turning marks initiate instabilities, but that they transition to those caused by the polarization of the light. The very center, magnified further on the right image, where the center is very smooth except for the few redeposited nanoparticles.

Pits were also machined into a titania aerogel that was approximately $1.8 \mathrm{~g} / \mathrm{cm}^{3}$ in density. The goal of the work was to show that hemispherical pits could be made using USP laser ablation. Figure 10 shows two pits ablated at $1 \mathrm{~J} / \mathrm{cm}^{2}$, which was really too high of a fluence for this material. A thin layer of remelt is shown in both pits shown. The only difference between the pits is that the deeper pit was the result of more ablation pulses. The promising aspect is that in the deeper hole relatively unaffected material can be observed around the edges of the hole where the fluence is low. Future experiments are being planned to revisit this at much lower, near threshold fluences. The goal is to achieve material removal without causing densification or the generation of boundaries due to remelt, which would not be desireable for the HEDP experiments.
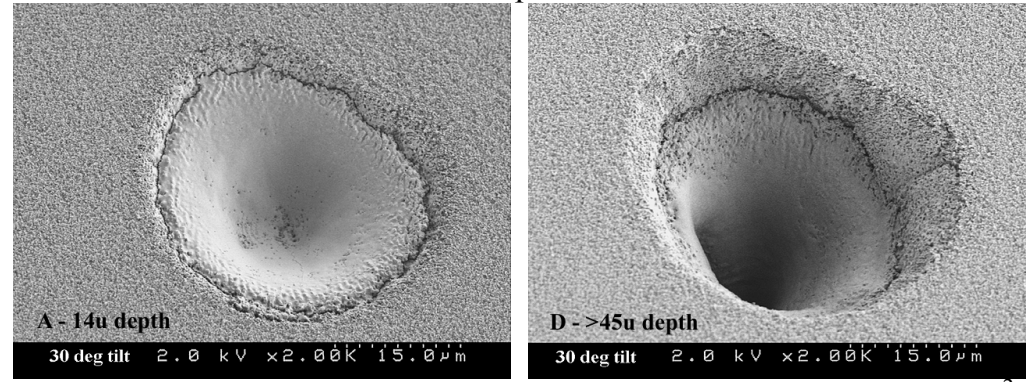

Figure 10. Pits in titania aerogel machined by $820-\mathrm{nm}, 100$-fs Ti:Sapphire laser using $1 \mathrm{~J} / \mathrm{cm}^{2}$.

The excimer surface sculpting of polymers was performed using polyimide. The system was used to sculpt moguls onto a polyimide surface using a projection of an illuminated round mask. This was done by precisely accelerating and decelerating a linear stage to raster out the pattern onto the surface. The results are shown in Figure 11 that shows the surface profile of the ablated surface. Smoother mogul surfaces have been generated by others, however these results were accomplished by using comb-like masks and ablating onto planar targets. Since this work will have to be transitioned to very small cylindrical and spherical geometries where relatively complex mask projection illumination is not easy, these single-point results appear promising. The results in Figure 11 have not been optimized, but they show promise that single point ablation can create surfaces of a desired mogul or dimpled profile on arbitrary shapes.

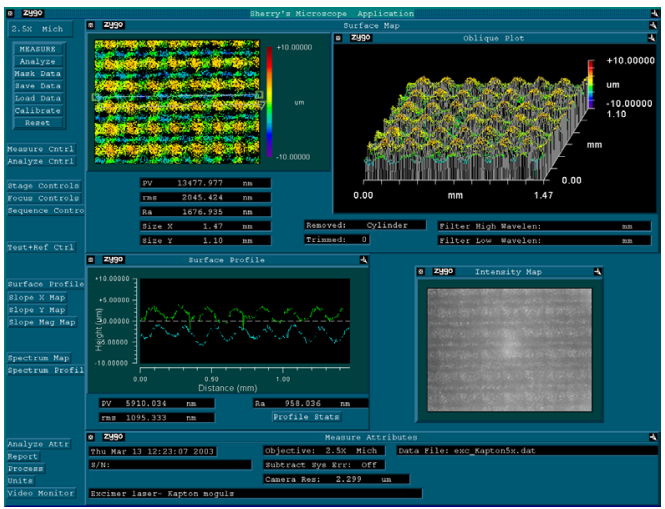

Figure 11. A two-dimension mogul field ablated on polyimide using a single point excimer ablation 


\subsubsection{Hole Drilling}

Holes were drilled in beryllium foils $(125-\mu \mathrm{m}$ thick) and thinner walled spheres for burst strength testing. Figure 12 shows a typical hole that is drilled into a beryllium foil. This produced the diameter that we desired and an aspect ratio between 30 and 40 . The material for the targets is significantly different in microstructure because it is made by sputter beryllium onto a plastic mandrel. We have demonstrated drilling of holes into this target material, as shown in Figure 13. An SEM image of the entire capsule is shown, the hole is present but is not easily discernible. The drilled hole is shown next to the capsule, and it is less than $5 \mu \mathrm{m}$ in diameter.
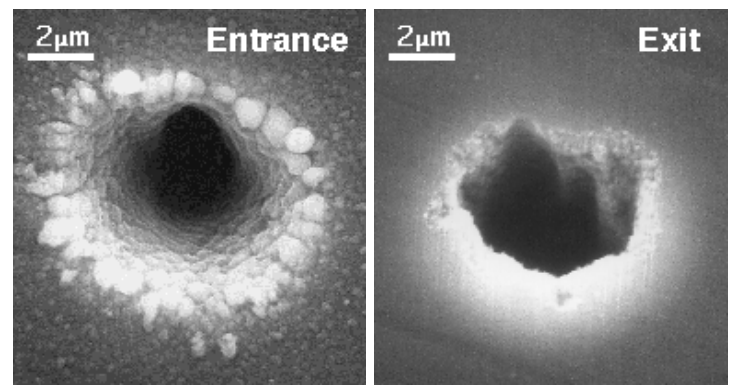

Figure 12. SEM images of 3 to $4-\mu \mathrm{m}$ diameter holes drilled in $125-\mu \mathrm{m}$ thick Beryllium foil
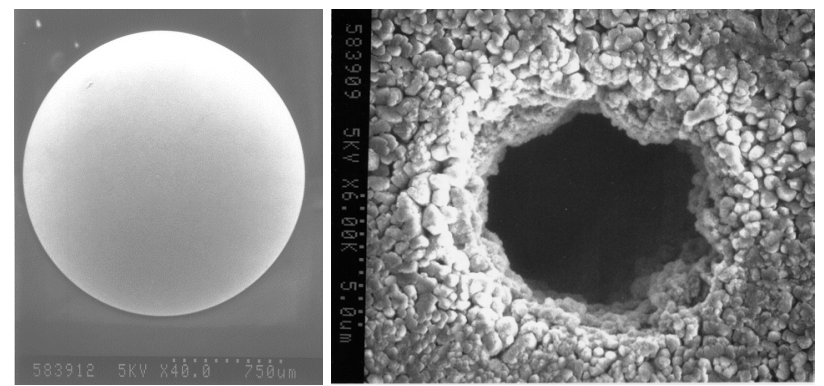

Figure 13. SEM image of a hole drilled into a beryllium capsule, the hole is barely visible in the image on the left.

\section{CONCLUSION}

This work has shown that the models of USP laser ablation of a surface agrees closely with the experimental evidence, and can allow the exploration of various regimes to achieve the desired results of very precise and smooth ablation of materials. This includes maintaining surface profiles of desired tolerances and RMS values below $100-$ $\mathrm{nm}$, RMS. These results are being used by HEDP and ICF scientists to design future experiments that allow tighter tolerances, and enabling them to have better control of the interface surfaces used in these targets. It will also help them to pick materials that, while potentially equivalent to others from a modeling standpoint, are easier and cheaper to machine and work with.

\section{REFERENCES}

[1] Li, C. K, Seguin, F. H., Frenje, J. A., Kurebayashi, S. and Petrasso, R. D. "Effects of Fuel-Shell Mix upon Direct-Drive, Spherical Implosions on OMEGA”, Phys Rev Let 89:165002, 2002.

[2] Robey, H. F., Kane, J. O, Remington, B. A., Drake, R. P., Hurricane, O. A., Louis, H., Wallace, R. J., Knauer, J., Keiter, P., Arnett, D. and Ryutov, D. D. "An experimental testbed for the study of hydrodynamic issues in supernovae," Physics of Plasmas 8:5, 2446-2453 (2001).

[3] Remington, B. A., Weber, S. V., Marinak, M. M., Haan, S. W., Kilkenny, J. D., Wallace, R. J., and Dimonte, G. "Single-mode and Multimode Rayleigh-Taylor experiments on Nova," Phys. Plasmas 2, 241 (1995).

[4] Jacobs, J. W. and Sheeley, J. M. "Experimental Study of Incompressible Richtmyer-Meshkov instability," Phys. Fluids 8, 405 (1996).

[5] Larson, J.T. "HYADES - A Radiation Hydrodynamics Code for Dense Plasma

Studies," in Radiative Properties of Hot Dense Matter, edited by W. Goldstein, C. Hooper, J. Gauthier, J. Seely and R. Lee (World Scientific, Singapore, 1991).

[6] EOS:SESAME Report on the Los Alamos Equation of state Library, Report No. LALP-83-4, T4 Group, Los Alamos National Laboratory, Los Alamos, 1983. 
[7] Mishin, Y., Mehl, M. J., and Papaconstantopoulos, D. A., Voter, A. F. and Kress, J. D. "Structural stability and lattice defects in copper: Ab initio, tight-binding, and embedded-atom calculations," Phys. Rev. B 63 (2001) 224106.

[8] Schafer, C., Urbassek, H. M., Zhigilei, L. V., Garrison, B. J. "Pressure-transmitting boundary conditions for molecular-dynamics simulations," Comp. Mat. Sci. 24, 421-429, (2002).

[9] Zhigilei, L. V. and Garrison, B. J. "Microscopic mechanisms of laser ablation of organic solids in the thermal and stress confinement irradiation regimes” J. Appl. Phys. 88, 1281-1298 (2000).

[10] Target fabricated by J. H. Satcher at Lawrence-Livermore National Laboratory, department of Chemistry and Materials Science.

[11] McEachern R., Alford, C., Cook, R., Makowiecki, D., Wallace, R. "Sputter-deposited Be ablators for NIF target capsules", Fusion Technology 31, 435-441, 1997.

This work was performed under the auspices of the U. S. Department of Energy by the University of California, Lawrence Livermore National Laboratory under Contract No. W-7405-Eng-48

\section{ABOUT THE AUTHORS}

Dr. Shirk received a M. S. in Materials Science and Engineering in 1996 and a Ph.D. in Mechanical Engineering in 1999, both from Iowa State University. He is an Engineer at Lawrence-Livermore National Laboratory. His main interests are in the development and use of laser technology for materials processing applications. Dr. Shirk is a member of the LIA.

Dr. Rubenchik received the Ph.D. degree in theoretical physics from the Institute of Nuclear Physics, Novosibirsk, in 1974, and the Dr. Sci. degree from the Space Research Institute, Moscow, in 1983. He is a Physicist at Lawrence Livermore National Laboratory. His main scientific interests are in physics of laser-matter interactions.

Dr. Gilmer is a physicist at Lawrence Livermore National Laboratory. He received his Ph.D. from the University of Virginia in 1963. He is a member of the APS, MRS, American Association for Crystal Growth, and Society for Industrial and Applied Mathematics.

Dr. Stuart is a physicist at Lawrence Livermore National Laboratory. He received his Ph.D. in Applied Science from the University of Davis in 1992.

Mr. Armstrong received his B.S. degree in Laser and Optical Engineering Technology from the Oregon Institute of Technology in 1996. He joined Lawrence Livermore National Laboratory immediately afterward and has focused mainly upon the engineering of optical, mechanical and control systems for femtosecond laser materials processing applications. Mr. Armstrong was a co-recipient of an R\&D 100 award for some of the early work in this field. He is a member of OSA and APS.

Mr. Nikitin is an Engineering Associate with 23 years of experience. He attended Naval Nuclear Engineering School. His interests are the development and deployment of x-ray diagnostics and machining of materials using the ArF excimer laser system to manufacture $x$-ray optics and laser targets.

Ms. Baker received an Associate Degree in Electron Microscopy from San Joaquin Delta College, Stockton CA. She is an Engineering Associate at Lawrence Livermore National Laboratory. Sherry's interest is in the application of state-of-the-art metrology techniques to the development of advanced x-ray optical components and systems. Her primary focus has been the characterization of optical components implemented in the development of extreme ultraviolet lithography (EUVL).

Dr. Mariella received his PhD in 1973 from Harvard University in Physical Chemistry and is the Director of the Center for Micro and Nano Technology at Lawrence-Livermore National Laboratory.

Approved for public release; further dissemination unlimited 